\title{
BMJ Open Views and experiences of seeking information and help for vitiligo: a qualitative study of written accounts
}

\author{
Emma Teasdale, ${ }^{1}$ Ingrid Muller, ${ }^{1,2}$ Amirah Abdullah Sani, ${ }^{1}$ Kim S Thomas, ${ }^{3}$ \\ Beth Stuart, ${ }^{1}$ Miriam Santer ${ }^{1}$
}

To cite: Teasdale E, Muller I, Abdullah Sani A, et al. Views and experiences of seeking information and help for vitiligo: a qualitative study of written accounts. BMJ Open 2018;8:e018652. doi:10.1136/ bmjopen-2017-018652

- Prepublication history for this paper is available online. To view these files, please visit the journal online (http://dx.doi. org/10.1136/bmjopen-2017018652).

Received 13 July 2017 Revised 6 December 2017 Accepted 8 December 2017

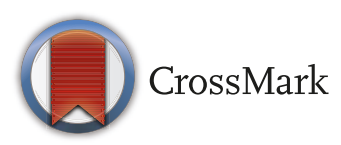

${ }^{1}$ Primary Care and Population Sciences, Faculty of Medicine University of Southampton,

Southampton, UK

${ }^{2}$ Academic Unit of Psychology, Faculty of Social and Human

Sciences, University of Southampton, Southampton, UK

${ }^{3}$ Centre for Evidence Based Dermatology, University of Nottingham, Nottingham, UK

Correspondence to

Dr Emma Teasdale;

e.j.teasdale@soton.ac.uk

\section{ABSTRACT}

Objectives Vitiligo is a relatively common autoimmune condition causing loss of skin pigment. Around 1 in 100 people in the UK develop vitiligo. It can have a significant impact on quality of life for many of those affected. How people access information and help for vitiligo may influence how they manage such impact. We aimed to explore people's views and experiences of seeking health information and help for vitiligo.

Design Qualitative analysis of free-text responses to four open-ended questions in an online survey.

Setting Online survey conducted in the UK between February and March 2016.

Participants A survey link was emailed to 675 members of The Vitiligo Society, a UK-based charity providing information and support for people with vitiligo. One hundred and sixty-one members responded to the survey (24\%)

Results Many participants wrote extensive free text, often reporting frustration with help-seeking. They perceived general practitioners (GP) as their primary source of advice but felt that GPs had low awareness of available treatments. Where GPs appeared sympathetic or signposted towards further information this was appreciated, even where people felt their GP had not seemed knowledgeable. Many felt that vitiligo was dismissed by health professionals including GPs and dermatologists as 'cosmetic', which upset those who experienced substantial impact. Participants expressed concerns about the credibility of online information on vitiligo and the need for reliable, detailed information, as well as a desire for support with managing its psychosocial impact.

Conclusions Information and help-seeking needs of people with vitiligo currently appear to be poorly met, even among members of The Vitiligo Society, who are likely to have received more information than others. People with vitiligo would welcome greater health professional awareness of available vitiligo treatments. Acknowledging the psychosocial impacts of vitiligo and signposting towards credible information are also welcomed.

\section{INTRODUCTION}

Vitiligo is an autoimmune disease where pale white patches progressively develop on the skin due to loss of pigment. Worldwide prevalence ranges from $0.5 \%$ to $2 \%$ and around

\section{Strengths and limitations of this study}

- We employed an efficient yet relatively underused method of qualitative data collection to explore an under-researched topic area.

- Conducting an exploratory qualitative analysis of the free-text comments within a quantitative survey allowed us to gain novel and valuable insights into, and a greater understanding about, the experiences of people with vitiligo in seeking information and treatment about their condition.

- A limitation of this study was our sampling method. Members of The Vitiligo Society may not be representative of all people with vitiligo, which limits the transferability of our findings. Participants in this study could have had greater impact from vitiligo, have been more unsatisfied with the information they had received, or more engaged with seeking treatment and information than other people with vitiligo.

- Also most of the participants in this study were over 45 , which is likely to be unrepresentative of the whole population of people with vitiligo as the condition commonly appears before the age of 20 .

1 in 100 people in the UK develop vitiligo. ${ }^{12}$ The appearance altering nature of vitiligo can have a significant impact on quality of life, particularly in terms of increased anxiety, lowered self-esteem and relationship difficulties, and it may also negatively affect self-confidence and body image. ${ }^{3} 4$ Most vitiligo develops before the age of 20 and may have a profound impact on confidence and selfimage at a key time in social development. ${ }^{5}$

The evidence base for vitiligo treatment is currently poor. ${ }^{6}$ A European guideline has suggested that early treatment of small lesions of recent-onset and childhood vitiligo should be treated with a combination of phototherapy and topical agents, such as topical corticosteroids or topical calcineurin inhibitors. ${ }^{7}$ Such treatments are less likely to be used if people with vitiligo do not consult 
health professionals or do not receive appropriate advice from health professionals.

Previous research has highlighted the psychosocial impact of vitiligo and the coping strategies adopted by people living with vitiligo. A survey carried out among members of The Vitiligo Society in 2010 showed that over half $(56.6 \%)$ of respondents felt that vitiligo moderately or severely affected their quality of life. ${ }^{8}$ One qualitative interview study carried out with seven white British women reported a continuous struggle using avoidance, concealment and cognitive strategies to try to deal with the impact of vitiligo. ${ }^{9}$ Another qualitative interview study was conducted with seven British women of South Asian descent. All had experienced stigmatisation to some extent. Avoidance and concealment were commonplace, as was a profound impact on their relationships and sense of identity. ${ }^{10}$

How people access and make sense of information, and seek help for vitiligo, is likely to influence how they cope with the psychosocial impacts, but there is relatively little research on people's experiences of obtaining information and treatment for vitiligo. Previous quantitative research suggests that people with vitiligo typically want to find a lasting effective treatment but they appear to have difficulty accessing treatment and feel that their condition is dismissed as 'trivial' or 'cosmetic' ${ }^{11}$ Members of The Vitiligo Society have reported that they obtain information about vitiligo from non-medical sources, such as The Vitiligo Society $(83 \%)$ and the internet $(25 \%)$ rather than from dermatologists (13\%) and general practitioners (GP) (7\%). ${ }^{8}$ Surprisingly few had obtained advice from GPs or dermatologists. Further research is needed to explore whether this relates to difficulties in accessing advice or whether people actively choose to obtain advice elsewhere.

Qualitative methods are well suited to exploring a topic in-depth from the perspective of a particular group of people. Qualitative research that seeks to explore people's experiences of obtaining information about vitiligo and their help-seeking behaviour could help determine whether people with vitiligo expect GPs or dermatologists to advise them about vitiligo or whether they generally seek information from charities or the internet instead of, or in addition to, consulting. This in turn would enable better understanding of how GPs and dermatologists can best support people with vitiligo in terms of information provision and/or signposting to reliable resources. Given the potential insights into people's experiences to be gained by using qualitative methods, ${ }^{12}$ we incorporated open-ended questions into a quantitative survey about information and help-seeking behaviour in people with vitiligo. We aimed to explore views and experiences of seeking information and help for vitiligo through analysis of the written accounts of survey respondents.

\section{METHODS}

This study sought to qualitatively analyse the free-text comments within a quantitative survey that focused on exploring sources of information for vitiligo, experiences of treatment and information needs. This qualitative approach has been previously used in other health-related research. ${ }^{13-15}$

\section{Main survey}

An anonymous online survey of people with vitiligo was conducted in the UK in February 2016 (response rate $161 / 675(24 \%))$. We sought participants through The Vitiligo Society partly because this had been a successful route for previous research, ${ }^{8}$ and also because informal advice suggested vitiligo might not be well coded in primary care so recruiting through this route would be problematic. Details about the study and a link to the online survey were emailed to 675 members of The Vitiligo Society. A reminder email was sent out after 2 weeks. The survey link was also made available on The Vitiligo Society web site from February to March 2016. Participants provided online informed consent prior to accessing the survey. In total, 161 people completed some or all of the survey questions and were included in the analysis (table 1). Quantitative survey findings regarding sources of information and help-seeking were similar to those previously reported. ${ }^{8}$ This paper focuses on the rich qualitative data obtained from the free-text responses to open-ended questions in the survey.

\section{Qualitative component}

The online survey included four open-ended questions, which allowed participants to respond in more detail (table 2). The survey data set was imported into NVivo V.10 and responses to the open-ended questions collated for analysis. A total of 495 responses to the open-ended questions (20728 words) were received from 158 of the 161 survey respondents (98\% of respondents completed the free-text questions in addition to the other survey questions). Responses to the open-ended questions ranged from brief comments (one phrase or sentence) to more detailed accounts (a couple of paragraphs). Question 3 (informational needs of people with vitiligo) received the most responses and question 2 (experiences of seeking treatment for vitiligo) received the most detailed comments.

\section{Data analysis}

An inductive thematic analysis was conducted to explore people's experiences of seeking information and help for vitiligo. ${ }^{16}$ One author (ET) read the comments several times to achieve familiarisation with the data and codes were applied line by line. Codes were derived inductively from the data and grouped together to produce an initial coding frame. Codes were compared to identify similarities and differences. A detailed coding manual (containing code names, code descriptions and data excerpts) was created to ensure transparent and systematic coding of 
Table 1 Survey respondents' characteristics

\begin{tabular}{|c|c|c|}
\hline & $\begin{array}{l}\text { Number of } \\
\text { participants } \\
n=161\end{array}$ & $\begin{array}{l}\text { Percentage } \\
(\%)\end{array}$ \\
\hline Answered for themselves & 151 & 93.8 \\
\hline $\begin{array}{l}\text { Answered on behalf of } \\
\text { other person }\end{array}$ & 4 & 2.5 \\
\hline Missing & 3 & 3.7 \\
\hline \multicolumn{3}{|l|}{ Gender } \\
\hline Male & 55 & 34.1 \\
\hline Female & 98 & 60.9 \\
\hline Missing & 8 & 5.0 \\
\hline \multicolumn{3}{|l|}{ Age (years) } \\
\hline $5-16$ & 2 & 1.2 \\
\hline $16-25$ & 8 & 5.0 \\
\hline $26-45$ & 27 & 16.8 \\
\hline $46-65$ & 73 & 45.3 \\
\hline $65+$ & 45 & 28.0 \\
\hline Missing & 6 & 3.7 \\
\hline \multicolumn{3}{|l|}{ Portion of skin affected } \\
\hline $\begin{array}{l}\text { Just a few patches } \\
(0 \%-10 \%)\end{array}$ & 35 & 21.7 \\
\hline $\begin{array}{l}\text { A fair amount (10\%- } \\
25 \%)\end{array}$ & 41 & 25.5 \\
\hline Quite a lot (25\%-50\%) & 33 & 20.5 \\
\hline Very much (50\%-80\%) & 25 & 15.5 \\
\hline $\begin{array}{l}\text { All or nearly all (more } \\
\text { than } 80 \% \text { ) }\end{array}$ & 19 & 11.8 \\
\hline Missing & 8 & 5.0 \\
\hline \multicolumn{3}{|l|}{ Skin colour } \\
\hline Fair & 67 & 41.6 \\
\hline Medium & 75 & 46.6 \\
\hline Dark & 10 & 6.2 \\
\hline Very dark & 1 & 0.6 \\
\hline Missing & 8 & 5.0 \\
\hline \multicolumn{3}{|l|}{ Family with vitiligo } \\
\hline Yes & 72 & 44.7 \\
\hline No & 82 & 50.9 \\
\hline Missing & 7 & 4.4 \\
\hline \multicolumn{3}{|l|}{ Living } \\
\hline UK & 147 & 91.3 \\
\hline Europe & 1 & 0.6 \\
\hline Elsewhere & 5 & 3.1 \\
\hline Missing & 8 & 5.0 \\
\hline
\end{tabular}

the data. Codes and theme/subtheme definitions were discussed with recourse to the data, and iteratively developed by three members of the research team (ET, MS and IM) to offer diverse inferences and interpretation of the data. A negative case analysis was carried out to ensure that all data were taken into account rather than just selecting data that fitted with the authors' viewpoint. Data saturation could not be determined due to the nature of the method of data collection. Using NVivo enabled a detailed audit trail to be maintained.

\section{RESULTS}

The majority of survey respondents $(n=158,98 \%)$ completed the open-ended questions, suggesting that those who responded to the survey were highly motivated to provide written feedback on their experiences of seeking help and information for vitiligo. Analysis of these data highlighted three themes: (1) experiences of consulting health professionals for vitiligo; (2) seeking information about vitiligo; and (3) perceptions and experiences of vitiligo treatments. These themes are explored in detail below. All quotes are labelled with a participant ID, gender and age.

\section{Experiences of consulting health professionals for vitiligo}

A sense of dissatisfaction and feeling unsupported was apparent in participants' reports about their experiences of consulting health professionals for vitiligo. Participants often seemed disappointed by the response they had received from GPs, who were viewed as their primary source of contact and advice. A common perception among participants was that GPs are not generally aware of, or particularly knowledgeable about, vitiligo and as such can only provide limited information and advice to patients.

None of the GPs I have spoken to have ever offered advice about Vitiligo. They seem to be somewhat in the dark about the problem. (Participant 36, male, over 65 years)

Where health professionals appeared sympathetic or where signposting towards further information was offered this was appreciated, even where people felt their GP had not been knowledgeable.

I have had vitiligo for about 35 years. I am now 70 years old. I have found that, with very few exceptions, GPs know little about the condition. One of my GPs (who 'half-retired' a few years ago) was always interested in learning more and asked me if I could provide some information on vitiligo. I gave her some printed material and she was grateful; I know that she read everything which I provided. (Participant 110, male, over 65 years)

Many participants felt they had just been left to 'get on with it' and learn to accept and manage their vitiligo on their own. For some participants this led them to seeking out alternative treatments not available in the UK. Another dominant perspective in this study was the sense that vitiligo is not taken seriously by medical professionals, both GPs and dermatologists. Many participants felt that their vitiligo was dismissed by health professionals 


\section{Questions}

Q1. We would be very interested to learn more about your experience of getting information for vitiligo. If you are happy to, please can you tell us some more about this?

Q2. We would be very interested to learn more about your experience of getting treatment for vitiligo. If you are happy to, please can you tell us some more about this?

Q3. Are there any aspects of vitiligo that you feel you would like to know more about? 148 (94)

Q4. Is there anything you would like to say that you feel has not been covered already? 102 (65)

\section{Number of} responses to each question (\%)

$142(90)$

$103(65)$
Total number

of words per question as something inconsequential, that is, purely cosmetic, which was upsetting to those experiencing substantial psychosocial impact.

I started my first signs of Vitiligo 30 years ago at the age of 40 . I went to my GP who referred me to a consultant. The consultant proved to be absolutely useless in providing any information. He just took one look at me, told me it was something called Vitiligo and told me that it would most likely get worse but wouldn't kill me!!! (Participant 71, female, over 65 years)

I noticed most doctors don't know much about vitiligo and really don't seem to care. I felt they had no clue about what a person with vitiligo goes through, Since it's not a matter of life or death, your expected to just accept it. I was very disappointed every time, always left feeling hopeless. I think they should have more to offer, be sympathetic \& at least try different things. (Participant 138, female, 46-65 years)

\section{Seeking information about vitiligo}

Participants commonly reported seeking information from other sources, predominantly online resources, as a result of receiving limited information from health professionals. The Vitiligo Society was viewed as a helpful and trustworthy source of information for participants but many expressed doubts about the reliability and credibility of other online resources about vitiligo. A common concern expressed by the participants related to the credibility of online information on possible treatments for vitiligo. In particular, they expressed doubts about the commercial interests of the web sites and about being able to determine the evidence base (safety and efficacy) of certain products and procedures.

I find it very difficult to find clear and consistent advice on possible treatments for Vitiligo. There are a lot of commercially orientated websites promoting a variety of herbal cures etc but limited proper evidence based medical guidance. (Participant 40, male, 26-45 years)

The only problem when searching for vitiligo sites is that some websites eventually lead into selling some sort of product which is the new 'best treatment'. You find that because you are motivated to find some sort of treatment you end up almost believing the hype that they sell. (Participant 139, male, 46-65 years)

A desire for detailed, reliable information about the causes of vitiligo, its progression and possible treatments was widely expressed. Some participants felt it important that such information should come directly from health professionals or other resources perceived to be trustworthy such as NHS Choices. Participants also expressed a desire for information and advice about managing the psychosocial impact of living with vitiligo, particularly advice on learning to cope with social situations and people's reactions to their altered appearance, and how to explain the condition to others.

I developed vitiligo 42 years ago when I was 2 . The GP referred me to a very good dermatologist who explained things clearly as well as, crucially, how to sensibly explain it to other children. (Participant 11, female, 26-45 years)

I would like more information on the cause of it and how to deal with being so self-conscious about itit does affect confidence and forming relationships. (Participant 130, female, 26-45 years)

\section{Perceptions and experiences of vitiligo treatments}

Experiences of vitiligo treatments expressed by participants were very mixed. While some participants expressed positive experiences of treatment, particularly ultraviolet (UV) light treatment, many participants appeared to have more negative experiences of seeking and receiving treatment for their vitiligo. A commonly expressed view was that vitiligo treatment is not routinely offered or is poorly available, especially on the NHS, and is limited to particular geographical locations.

We decided to have light treatment. My main concern was the Vitiligo on my face. The treatment worked well but a few years later it appeared elsewhere on my face so I had more treatment that also worked well. The spread of vitiligo has slowed to almost a 
stop apart from a couple of new areas on my face. (Participant 74, male, 46-65 years)

There is very little treatment for Vitiligo in the UK. I am at present involved with the Hi-Light trial run by the Univ of Nottingham. This is the first time I have been able to obtain any treatment. (Participant 102, male, over 65 years)

Many participants, who had received treatments such as steroid creams and light treatment for their vitiligo, felt they were ineffective in the long term as the treatments had been unsuccessful in maintaining any areas of repigmentation. Participants also reported the perceived difficulties and potential side effects of vitiligo treatment such as concerns about skin thinning with steroid creams, time-consuming nature and inconvenience of hospital-based UV light treatment and the physical side effects of light treatment such as nausea and headaches.

I was referred to Dermatology and was prescribed PUVA treatment. This helped for a while, but became so time consuming. Progress was very slow. I stopped the treatment and any areas of repigmentation soon reverted back to white skin. So disappointing. (Participant 156, female, 46-65 years)

As well as mixed experiences of vitiligo treatments, there were mixed attitudes towards the need to seek vitiligo treatments in the first instance. Some participants reported less experience of treatments because they had not sought these out and had 'accepted' their vitiligo, even though it may have had a profound impact on their lives.

I think that vitiligo is a relatively unknown condition. I have never been contacted about treatment or had it offered to me despite having had the condition for many years. You are very much left to get on with it. I can imagine that some people suffer psychologically and I wonder what support they get. I have come to terms with my patches and embrace them rather than disguise them but it can feel quite disheartening at times and I would rather not have them given the choice. (Participant 58, female, 46-65 years)

\section{DISCUSSION}

We found predominantly negative experiences of help-seeking, concerns about the credibility of online information and the need for detailed, reliable vitiligo information. Frustration with challenges to helpseeking was often reported, particularly perceived lack of awareness of available treatments among GPs. Many felt that vitiligo was often dismissed by health professionals as 'cosmetic', which was upsetting to those experiencing substantial psychosocial impact. Where GPs appeared sympathetic or where signposting towards further information was offered this was appreciated, even where people felt their GP had not seemed knowledgeable. There were very mixed views about the need for treatment and a desire for support with managing the psychosocial impact of vitiligo.

This study provides valuable insights into the experiences of people with vitiligo in seeking information and treatment about their condition. We employed an efficient yet relatively underused method of qualitative data collection to explore an under-researched topic area. Given the relative anonymity of providing written responses in an online survey, these data may have been more candid than data collected in face-to-face research settings. However, it was not possible in this context to ask participants to expand on or clarify their free-text responses. Being able to clarify and explore further views and experiences of patients with vitiligo in a qualitative interview may have generated richer data that would have enabled a more in-depth qualitative analysis.

Our findings also need to be seen in the context of our sampling method. People with vitiligo who are members of The Vitiligo Society may not be representative of all people with vitiligo. Members of The Vitiligo Society pay an annual membership fee (£26), which may be a barrier to membership for some people. The response rate for our survey, which was carried out entirely online, was lower than the previous survey among The Vitiligo Society members, ${ }^{8}$ which was carried out by post. We did not compare responders with non-responders and so we cannot be sure that our participants are different in some way from those who did not respond to the survey. This may mean that participants in this study had greater impact from vitiligo, were more unsatisfied with the information they had received, or were more engaged with seeking treatment and information than other people with vitiligo, and limits the transferability of the findings

Most of the participants in this study were over 45 , which is likely to be unrepresentative of the whole population of people with vitiligo as the condition commonly appears before the age of $20 .{ }^{17}{ }^{18}$ It is likely that most of the participants had had vitiligo for many years and their experience may differ from those more recently diagnosed. Furthermore, by sampling through a patient support group we may have been more likely to receive accounts from people whose needs had not been met by health services, although it is notable that this group still felt that they had many unanswered questions and that they found online information difficult to navigate.

The feeling that vitiligo is dismissed by health professionals as 'cosmetic' or 'trivial' shows that this is an ongoing concern for people, in common with previous reports, ${ }^{9} 10$ and also in line with other skin conditions. ${ }^{19}{ }^{20}$ We found that people can find it difficult to judge the credibility of online information on vitiligo, as in other conditions, ${ }^{21}$ but it seems surprising in this context where participants are all members of a relevant support group. We also found that participants 
Table 3 Online resources for people with vitiligo

\begin{tabular}{ll}
\hline Web site & URL \\
\hline NHS Choices & http://www.nhs.uk/conditions/Vitiligo/Pages/Introduction.aspx \\
& Links to the official web site of the National Health Service in England. Their section on vitiligo provides \\
& information and advice about vitiligo symptoms, causes, diagnosis and treatments. \\
Patient.co.uk & http://patient.info/health/vitiligo-leaflet \\
& Links to a general medical information and support web site providing information and advice about \\
& vitiligo symptoms, causes and treatments \\
The Vitiligo Society & $\begin{array}{l}\text { http://www.vitiligosociety.org.uk/ } \\
\text { Links to The Vitiligo Society web site, a UK-based charity providing information and support for people } \\
\text { with vitiligo through their regularly updated web site and newsletter, Dispatches }\end{array}$ \\
\hline
\end{tabular}

still generally view health professionals as an important source of information, which has been noted in other studies. ${ }^{22}$ Again, it seems surprising that our participants, who were members of a support group and many of whom reported negative experiences of consulting, still wanted health professionals to be the main source of information. Our finding that people are keen for support with managing the psychosocial impact of living with vitiligo was also found in a recent James Lind Alliance Priority Setting Partnership in the UK, which identified the need for further research into the effectiveness of psychological support for people with vitiligo, either on its own or together with other treatments. ${ }^{23}$ The importance of providing people with skin diseases with access to a range of supportive services including psychological support was also acknowledged in a recent report of the All Party Parliamentary Group on Skin. ${ }^{24}$

Constraints on access to specialists through primary care 'gatekeeping' or 'managed care' may be associated with lower patient satisfaction, ${ }^{25}$ and respondents in this study expressed frustration with generalist services, perceiving them to have a low level of knowledge about their condition. It is not completely clear whether this represents lack of awareness among GPs of available treatments for vitiligo, or lack of provision of services into which they can refer. Access to vitiligo services/ treatment is becoming increasingly difficult in many areas of the UK, which is likely to increase people's frustration with help-seeking and may exacerbate the psychosocial impact experienced by many people with vitiligo.

This paper highlights opportunities for further qualitative and quantitative research exploring and examining the needs of people with vitiligo, particularly younger people with vitiligo and those with a recent diagnosis. There is also a paucity of comparative evidence for effective treatments for vitiligo. In addition to quantitative research examining the effectiveness of treatments, greater evidence is also needed on how best to address the psychosocial impact of living with vitiligo. In this study, people's support needs included advice on managing social situations and explaining the condition to others, learning how to use camouflage, as well as clear and up-to-date information about the pros and cons of available treatments. Further research to develop and test education and psychological support interventions that seek to address the information and support needs of people with vitiligo is likely to be valuable. In an era where people are increasingly obtaining health information online,${ }^{26}$ it may be that education and psychological support interventions delivered online may be beneficial.

In conclusion, more can be done to meet the information and help-seeking needs of people with vitiligo. People with vitiligo would welcome greater health professional awareness of the possibility of treating vitiligo, yet recognise that generalist services may not always be able to offer this. People who had been directed towards reliable information were very grateful for this and signposting is viewed as a key role of GPs, which remain the primary source of information for many. Fortunately, good online resources for people with vitiligo are available (table 3). Signposting people with vitiligo towards credible online resources and acknowledging and exploring the potential impact of the condition may allay people's perceptions that distress caused by the condition is not taken seriously.

Contributors MS, IM and KST designed the study. AAS collected the data under the supervision of MS and BS. ET, IM, BS and MS made substantial contributions to the analysis and interpretation of data. ET drafted the manuscript. IM, MS, BS, AAS and KST critically reviewed and revised the manuscript. All authors gave the final approval of the version to be published.

Funding This research received no specific grant from any funding agency in the public, commercial or not-for-profit sectors.

Competing interests None declared.

Patient consent Detail has been removed from this case description/these case descriptions to ensure anonymity. The editors and reviewers have seen the detailed information available and are satisfied that the information backs up the case the authors are making.

Ethics approval Faculty of Medicine Research Ethics Committee, University of Southampton.

Provenance and peer review Not commissioned; externally peer reviewed.

Data sharing statement This is a qualitative study and therefore the data generated are not suitable for sharing beyond that contained within the report. Further information can be obtained from the corresponding author.

Open Access This is an Open Access article distributed in accordance with the Creative Commons Attribution Non Commercial (CC BY-NC 4.0) license, which 
permits others to distribute, remix, adapt, build upon this work non-commercially, and license their derivative works on different terms, provided the original work is properly cited and the use is non-commercial. See: http://creativecommons.org/ licenses/by-nc/4.0/

(C) Article author(s) (or their employer(s) unless otherwise stated in the text of the article) 2018. All rights reserved. No commercial use is permitted unless otherwise expressly granted.

\section{REFERENCES}

1. Osinubi O, Grainge MJ, Hong L, et al. The prevalence of psychological co-morbidity in people with vitiligo: a systematic review and meta-analysis. Br J Dermatol 2017.

2. Zhang Y, Cai Y, Shi M, et al. The prevalence of vitiligo: a metaanalysis. PLoS One 2016;11:e0163806.

3. Porter JR, Beuf AH, Lerner A, et al. Psychosocial effect of vitiligo: a comparison of vitiligo patients with "normal" control subjects, with psoriasis patients, and with patients with other pigmentary disorders. J Am Acad Dermatol 1986;15:220-4.

4. Radtke MA, Schäfer I, Gajur A, et al. Willingness-to-pay and quality of life in patients with vitiligo. Br J Dermatol 2009;161:134-9.

5. Silverberg JI, Silverberg NB. Quality of life impairment in children and adolescents with vitiligo. Pediatr Dermatol 2014;31:309-18.

6. Whitton ME, Pinart M, Batchelor J, et al. Interventions for vitiligo. Cochrane Database Syst Rev 2010:CD003263.

7. Taieb A, Alomar A, Böhm M, et al. Guidelines for the management of vitiligo: the European Dermatology Forum consensus. Br J Dermatol 2013;168:5-19.

8. Talsania N, Lamb B, Bewley A. Vitiligo is more than skin deep: a survey of members of the Vitiligo Society. Clin Exp Dermatol 2010;35:736-9.

9. Thompson AR, Kent G, Smith JA. Living with vitiligo: dealing with difference. Br J Health Psychol 2002;7(Pt 2):213-25.

10. Thompson AR, Clarke SA, Newell RJ, et al. Vitiligo linked to stigmatization in British South Asian women: a qualitative study of the experiences of living with vitiligo. Br J Dermatol 2010;163:481-6.

11. Ezzedine $\mathrm{K}$, Sheth V, Rodrigues M, et al. Vitiligo is not a cosmetic disease. J Am Acad Dermatol 2015;73:883-5.

12. Nelson PA. Getting under the skin: qualitative methods in dermatology research. Br J Dermatol 2015;172:841-3.
13. Bishop FL, Howick J, Heneghan C, et al. Placebo use in the UK: a qualitative study exploring GPs' views on placebo effects in clinical practice. Fam Pract 2014;31:357-63.

14. Corner J, Wagland R, Glaser A, et al. Qualitative analysis of patients feedback from a PROMs survey of cancer patients in England. BMJ Open 2013;3:e002316.

15. Richards $\mathrm{SH}$, Campbell JL, Walshaw E, et al. A multi-method analysis of free-text comments from the UK General Medical Council Colleague Questionnaires. Med Educ 2009;43:757-66.

16. Joffe H, Yardley L. Content and thematic analysis. In: Research Methods for Clinical and Health Psychology. London, UK: Sage, 2004:56-68.

17. Naik A. Vitiligo: compilation of clinico-epidemiological features in patients attending tertiary care government hospital, Thane. Australas Med J 2010;3:826-32.

18. Reghu R, James E. Epidemiological profile and treatment pattern of vitiligo in a tertiary care teaching hospital. Int J Pharm Sci 2011;3:137-41.

19. Magin PJ, Adams J, Heading GS, et al. Patients with skin disease and their relationships with their doctors: a qualitative study of patients with acne, psoriasis and eczema. Med J Aust 2009;190:62-4

20. Nelson PA, Chew-Graham CA, Griffiths CE, et al. Recognition of need in health care consultations: a qualitative study of people with psoriasis. Br J Dermatol 2013;168:354-61.

21. Santer M, Muller I, Yardley L, et al. 'You don't know which bits to believe': qualitative study exploring carers' experiences of seeking information on the internet about childhood eczema. BMJ Open 2015;5:e006339.

22. Sillence E, Briggs P, Harris PR, et al. How do patients evaluate and make use of online health information? Soc Sci Med 2007;64:1853-62.

23. Eleftheriadou V, Whitton ME, Gawkrodger DJ, et al. Future research into the treatment of vitiligo: where should our priorities lie? Results of the vitiligo priority setting partnership. Br J Dermatol 2011;164:no-36.

24. APPGS. 2013. The psychological and social impact of skin diseases on people's lives. A report of the all party parliamentary group on skin updated 2013 edition: London.

25. Greenfield G, Foley K, Majeed A. Rethinking primary care's gatekeeper role. BMJ 2016;354:i4803.

26. Mazanderani F, Powell J. Using the internet as a source of information about patients' experiences. Understanding and Using Health Experiences: Improving patient care, 2013:94-103. 